\title{
Social Network Games with Obligatory Product Selection
}

\author{
Krzysztof R. Apt \\ Centre for Mathematics and Computer Science (CWI), \\ ILLC, University of Amsterdam, The Netherlands \\ k.r.apt@cwi.nl
}

\author{
Sunil Simon \\ Centre for Mathematics and Computer Science (CWI) \\ s.e.simon@cwi.nl
}

\begin{abstract}
Recently, we introduced in [1] a model for product adoption in social networks with multiple products, where the agents, influenced by their neighbours, can adopt one out of several alternatives (products). To analyze these networks we introduce social network games in which product adoption is obligatory.

We show that when the underlying graph is a simple cycle, there is a polynomial time algorithm allowing us to determine whether the game has a Nash equilibrium. In contrast, in the arbitrary case this problem is NP-complete. We also show that the problem of determining whether the game is weakly acyclic is co-NP hard.

Using these games we analyze various types of paradoxes that can arise in the considered networks. One of them corresponds to the well-known Braess paradox in congestion games. In particular, we show that social networks exist with the property that by adding an additional product to a specific node, the choices of the nodes will unavoidably evolve in such a way that everybody is strictly worse off.
\end{abstract}

\section{Introduction}

Social networks became a huge interdisciplinary research area with important links to sociology, economics, epidemiology, computer science, and mathematics. A flurry of numerous articles, notably the influential [11], and books, e.g., [7, 3], helped to delineate better this area. It deals with many diverse topics such as epidemics, spread of certain patterns of social behaviour, effects of advertising, and emergence of 'bubbles' in financial markets.

Recently, we introduced in [1] social networks with multiple products, in which the agents (players), influenced by their neighbours, can adopt one out of several alternatives (products). To study the situation when the product adoption is obligatory we introduce here social network games in which product adoption is obligatory. An example of a studied situation is when a group of people chooses an obligatory 'product', for instance, an operating system or a mobile phone provider, by taking into account the choice of their friends. The resulting games exhibit the following join the crowd property:

the payoff of each player weakly increases when more players choose his strategy.

that we define more precisely in Subsection 2.3

The considered games are a modification of the strategic games that we recently introduced in [14] and more fully in [15], in which the product adoption was optional. The insistence on product selection leads to a different analysis and different results than the ones reported there. In particular, Nash equilibria need not exist already in the case when the underlying graph is a simple cycle. We show that one can determine in polynomial time whether for such social networks a Nash equilibrium exists. We prove that for arbitrary networks, determining whether a Nash equilibrium exists is NP-complete. We also show that for arbitrary networks and for networks whose underlying graph has no source nodes, determining whether the game is weakly acyclic is co-NP hard.

Gabriele Puppis, Tiziano Villa (Eds.): Fourth International Symposium on Games, Automata, Logics and Formal Verification EPTCS 119, 2013, pp. 180-193, doi 10.4204/EPTCS.119.16 (c) Krzysztof R. Apt \& Sunil Simon

This work is licensed under the Creative Commons Attribution License. 
The considered social networks allow us to analyze various paradoxes that were identified in the literature. One example is the paradox of choice first formulated in [13]. It has been summarised in [6, page 38] as follows:

The more options one has, the more possibilities for experiencing conflict arise, and the more difficult it becomes to compare the options. There is a point where more options, products, and choices hurt both seller and consumer.

The point is that consumers choices depend on their friends' and acquaintances' preferences.

Another example is a 'bubble' in a financial market, where a decision of a trader to switch to some new financial product triggers a sequence of transactions, as a result of which all traders involved become worse off.

Such paradoxes are similar to the renowned Braess paradox which states that in some road networks the travel time can actually increase when new roads are added, see, e.g., [12, pages 464-465] and a 'dual' version of Braess paradox that concerns the removal of road segments, studied in [4, 5]. Both paradoxes were studied by means of congestion games. However, in contrast to congestion games, Nash equilibria do not need to exist in the games we consider here. Consequently, one needs to rely on different arguments. Moreover, there are now two new types of paradoxes that correspond to the situations when an addition, respectively, removal, of a product can lead to a game with no Nash equilibrium.

For each of these four cases we present a social network that exhibits the corresponding paradox. These paradoxes were identified first in [2] in the case when the adoption of a product was not obligatory. In contrast to the case here considered the existence of a strongest paradox within the framework of [2] remains an open problem.

\section{Preliminaries}

\subsection{Strategic games}

A strategic game for $n>1$ players, written as $\left(S_{1}, \ldots, S_{n}, p_{1}, \ldots, p_{n}\right)$, consists of a non-empty set $S_{i}$ of strategies and a payoff function $p_{i}: S_{1} \times \cdots \times S_{n} \rightarrow \mathbb{R}$, for each player $i$.

Fix a strategic game $G:=\left(S_{1}, \ldots, S_{n}, p_{1}, \ldots, p_{n}\right)$. We denote $S_{1} \times \cdots \times S_{n}$ by $S$, call each element $s \in S$ a joint strategy, denote the $i$ th element of $s$ by $s_{i}$, and abbreviate the sequence $\left(s_{j}\right)_{j \neq i}$ to $s_{-i}$. Occasionally we write $\left(s_{i}, s_{-i}\right)$ instead of $s$.

We call a strategy $s_{i}$ of player $i$ a best response to a joint strategy $s_{-i}$ of his opponents if $\forall s_{i}^{\prime} \in$ $S_{i} p_{i}\left(s_{i}, s_{-i}\right) \geq p_{i}\left(s_{i}^{\prime}, s_{-i}\right)$. We call a joint strategy $s$ a Nash equilibrium if each $s_{i}$ is a best response to $s_{-i}$. Further, we call a strategy $s_{i}^{\prime}$ of player $i$ a better response given a joint strategy $s$ if $p_{i}\left(s_{i}^{\prime}, s_{-i}\right)>p_{i}\left(s_{i}, s_{-i}\right)$.

By a profitable deviation we mean a pair $\left(s, s^{\prime}\right)$ of joint strategies such that $s^{\prime}=\left(s_{i}^{\prime}, s_{-i}\right)$ for some $s_{i}^{\prime}$ and $p_{i}\left(s^{\prime}\right)>p_{i}(s)$. Following [10], an improvement path is a maximal sequence of profitable deviations. Clearly, if an improvement path is finite, then its last element is a Nash equilibrium. A game is called weakly acyclic (see [16, 9]) if for every joint strategy there exists a finite improvement path that starts at it. In other words, in weakly acyclic games a Nash equilibrium can be reached from every initial joint strategy by a sequence of unilateral deviations. Given two joint strategies $s$ and $s^{\prime}$ we write

- $s>s^{\prime}$ if for all $i, p_{i}(s)>p_{i}\left(s^{\prime}\right)$.

When $s>s^{\prime}$ holds we say that $s^{\prime}$ is strictly worse than $s$. 


\subsection{Social networks}

We are interested in strategic games defined over a specific type of social networks introduced in [1] that we recall first.

Let $V=\{1, \ldots, n\}$ be a finite set of agents and $G=(V, E, w)$ a weighted directed graph with $w_{i j} \in$ $[0,1]$ being the weight of the edge $(i, j)$. Given a node $i$ of $G$, we denote by $N(i)$ the set of nodes from which there is an incoming edge to $i$. We call each $j \in N(i)$ a neighbour of $i$ in $G$. We assume that for each node $i$ such that $N(i) \neq \emptyset, \sum_{j \in N(i)} w_{j i} \leq 1$. An agent $i \in V$ is said to be a source node in $G$ if $N(i)=\emptyset$. Given a (to be defined) network $\mathscr{S}$ we denote by source $(\mathscr{S})$ the set of source nodes in the underlying graph $G$.

By a social network (from now on, just network) we mean a tuple $\mathscr{S}=(G, \mathscr{P}, P, \theta)$, where

- $G$ is a weighted directed graph,

- $\mathscr{P}$ is a finite set of alternatives or products,

- $P$ is function that assigns to each agent $i$ a non-empty set of products $P(i)$ from which it can make a choice,

- $\theta$ is a threshold function that for each $i \in V$ and $t \in P(i)$ yields a value $\theta(i, t) \in(0,1]$.

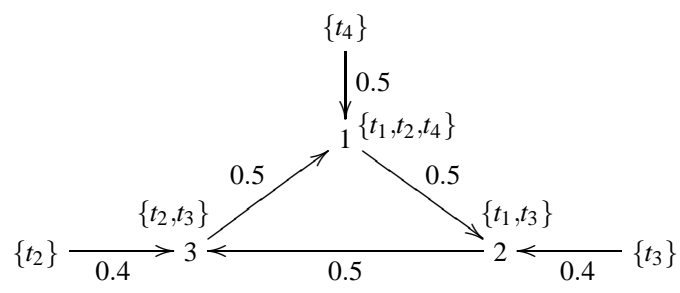

Figure 1: A social network

Example 1. Figure 1 shows an example of a network. Let the threshold be 0.3 for all nodes. The set of products $\mathscr{P}$ is $\left\{t_{1}, t_{2}, t_{3}, t_{4}\right\}$, the product set of each agent is marked next to the node denoting it and the weights are labels on the edges. Each source node is represented by the unique product in its product set.

Given two social networks $\mathscr{S}$ and $\mathscr{S}^{\prime}$ we say that $\mathscr{S}^{\prime}$ is an expansion of $\mathscr{S}$ if it results from adding a product to the product set of a node in $\mathscr{S}$. We say then also that $\mathscr{S}$ is a contraction of $\mathscr{S}^{\prime}$.

\subsection{Social network games}

Next, introduce the strategic games over the social networks. They form a modification of the games studied in [14, 15] in that we do not admit a strategy representing the fact that a player abstains from choosing a product.

Fix a network $\mathscr{S}=(G, \mathscr{P}, P, \theta)$. With each network $\mathscr{S}$ we associate a strategic game $\mathscr{G}(\mathscr{S})$. The idea is that the agents simultaneously choose a product. Subsequently each node assesses his choice by comparing it with the choices made by his neighbours. Formally, we define the game as follows:

- the players are the agents (i.e., the nodes),

- the set of strategies for player $i$ is $S_{i}:=P(i)$, 
- For $i \in V, t \in P(i)$ and a joint strategy $s$, let $\mathscr{N}_{i}^{t}(s):=\left\{j \in N(i) \mid s_{j}=t\right\}$, i.e., $\mathscr{N}_{i}^{t}(s)$ is the set of neighbours of $i$ who adopted in $s$ the product $t$.

The payoff function is defined as follows, where $c_{0}$ is some given in advance positive constant:

- for $i \in \operatorname{source}(\mathscr{S})$, $p_{i}(s):=c_{0}$,

- for $i \notin \operatorname{source}(\mathscr{S})$, $p_{i}(s):=\sum_{j \in \mathscr{N}_{i}^{t}(s)} w_{j i}-\theta(i, t)$, where $s_{i}=t$ and $t \in P(i)$.

In the first case we assume that the payoff function for the source nodes is constant only for simplicity. The second case of the payoff definition is motivated by the following considerations. When agent $i$ is not a source node, his 'satisfaction' from a joint strategy depends positively from the accumulated weight (read: 'influence') of his neighbours who made the same choice as him, and negatively from his threshold level (read: 'resistance') to adopt this product. The assumption that $\theta(i, t)>0$ reflects the view that there is always some resistance to adopt a product.

We call these games social network games with obligatory product selection, in short, social network games.

Example 2. Consider the network given in Example 1 and the joint strategy $s$ where each source node chooses the unique product in its product set and nodes 1,2 and 3 choose $t_{2}, t_{3}$ and $t_{2}$ respectively. The payoffs are then given as follows:

- for the source nodes, the payoff is the fixed constant $c_{0}$,

- $p_{1}(s)=0.5-0.3=0.2$,

- $p_{2}(s)=0.4-0.3=0.1$,

- $p_{3}(s)=0.4-0.3=0.1$.

Let $s^{\prime}$ be the joint strategy in which player 3 chooses $t_{3}$ and the remaining players make the same choice as given in $s$. Then $\left(s, s^{\prime}\right)$ is a profitable deviation since $p_{3}\left(s^{\prime}\right)>p_{3}(s)$. In what follows, we represent each profitable deviation by a node and a strategy it switches to, e.g., $3: t_{3}$. Starting at $s$, the sequence of profitable deviations $3: t_{3}, 1: t_{4}$ is an improvement path which results in the joint strategy in which nodes 1,2 and 3 choose $t_{4}, t_{3}$ and $t_{3}$ respectively and, as before, each source node chooses the unique product in its product set.

By definition, the payoff of each player depends only on the strategies chosen by his neighbours, so the social network games are related to graphical games of [8]. However, the underlying dependence structure of a social network game is a directed graph. Further, note that these games satisfy the join the crowd property that we define as follows:

Each payoff function $p_{i}$ depends only on the strategy chosen by player $i$ and the set of players who also chose his strategy. Moreover, the dependence on this set is monotonic.

The last qualification is exactly opposite to the definition of congestion games with player-specific payoff functions of [9] in which the dependence on the above set is antimonotonic. That is, when more players choose the strategy of player $i$, then his payoff weakly decreases.

\section{Nash equilibria}

The first natural question we address is whether the social network games have a Nash equilibrium. 


\subsection{Simple cycles}

In contrast to the case of games studied in [14] the answer is negative already for the case when the underlying graph is a simple cycle.

Example 3. Consider the network given in Figure 2, where the product set of each agent is marked next to the node denoting it and the weights are all equal and put as labels on the edges.

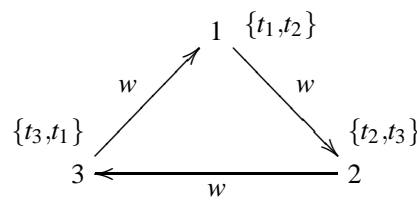

Figure 2: A simple cycle no Nash equilibrium

Let the thresholds be defined as follows: $\theta\left(1, t_{1}\right)=\theta\left(2, t_{2}\right)=\theta\left(3, t_{3}\right)=r_{1}$ and $\theta\left(1, t_{2}\right)=\theta\left(2, t_{3}\right)=$ $\theta\left(3, t_{1}\right)=r_{2}$ where $r_{1}>r_{2}$. We also assume that $w>r_{1}-r_{2}$. Hence for all $s_{2}$ and $s_{3}$

$$
p_{1}\left(t_{1}, s_{2}, t_{1}\right)>p_{1}\left(t_{2}, s_{2}, s_{3}\right)>p_{1}\left(t_{1}, s_{2}, t_{3}\right)
$$

and similarly for the payoff functions $p_{2}$ and $p_{3}$. So it is more profitable for player $i$ to adopt strategy $t_{i}$ provided its neighbour also adopts $t_{i}$.

It is easy to check that the game associated with this network has no Nash equilibrium. Indeed, here is the list of all the joint strategies, where we underline the strategy that is not a best response to the choice of other players: $\left(t_{1}, \underline{t_{2}}, t_{1}\right),\left(t_{1}, \underline{t_{2}}, t_{3}\right),\left(t_{1}, t_{3}, \underline{t_{1}}\right),\left(\underline{t_{1}}, t_{3}, t_{3}\right),\left(\underline{t_{2}}, t_{2}, t_{1}\right),\left(t_{2}, t_{2}, \underline{t_{3}}\right),\left(\underline{t_{2}}, t_{3}, t_{1}\right),\left(t_{2}, \underline{t_{3}}, t_{3}\right)$.

This example can be easily generalized to the case of an arbitrary simple cycle. Below, $i \oplus 1$ and $i \ominus 1$ stand for addition and subtraction defined cyclically over the set $\{1, \ldots, n\}$. So $n \oplus 1=1$ and $1 \ominus 1=n$. Indeed, consider a social network with $n$ nodes that form a simple cycle and assume that each player $i$ has strategies $t_{i}$ and $t_{i \oplus 1}$. Choose for each player $i$ the weights $w_{i \ominus 1 i}$ and the threshold function $\theta(i, t)$ so that

$$
w_{i \ominus 1 i}-\theta\left(i, t_{i}\right)>-\theta\left(i, t_{i \oplus 1}\right)>-\theta\left(i, t_{i}\right),
$$

so that (we put on first two positions, respectively, the strategies of players $i \ominus 1$ and $i$, while the last argument is a joint strategy of the remaining $n-2$ players)

$$
p_{i}\left(t_{i}, t_{i}, s\right)>p_{i}\left(t^{\prime}, t_{i \oplus 1}, s^{\prime}\right)>p_{i}\left(t_{i \ominus 1}, t_{i}, s^{\prime \prime}\right)
$$

where $t^{\prime}, s, s^{\prime}$ and $s^{\prime \prime}$ are arbitrary. It is easy to check then that the resulting social network game has no Nash equilibrium.

A natural question is what is the complexity of determining whether a Nash equilibrium exists. First we consider this question for the special case when the underlying graph is a simple cycle.

Theorem 4. Consider a network $\mathscr{S}$ whose underlying graph is a simple cycle. It takes $O\left(n \cdot|\mathscr{P}|^{4}\right)$ time to decide whether the game $\mathscr{G}(\mathscr{S})$ has a Nash equilibrium.

Proof. Suppose $\mathscr{S}=(G, \mathscr{P}, P, \theta)$. When the underlying graph of $\mathscr{S}$ is a simple cycle, the concept of a best response of player $i \oplus 1$ to a strategy of player $i$ is well-defined. Let

$$
R_{i}:=\left\{\left(t_{i}, t_{i \oplus 1}\right) \mid t_{i} \in P(i), t_{i \oplus 1} \in P(i \oplus 1), t_{i \oplus 1} \text { is a best response to } t_{i}\right\},
$$




$$
I:=\{(t, t) \mid t \in \mathscr{P}\}
$$

and let $\circ$ stand for the composition of binary relations.

The question whether $\mathscr{G}(\mathscr{S})$ has a Nash equilibrium is then equivalent to the problem whether there exists a sequence $a_{1}, \ldots, a_{n}$ such that $\left(a_{1}, a_{2}\right) \in R_{1}, \ldots,\left(a_{n-1}, a_{n}\right) \in R_{n-1},\left(a_{n}, a_{1}\right) \in R_{n}$. In other words, is $\left(R_{1} \circ \cdots \circ R_{n}\right) \cap I$ non-empty?

To answer this question we first construct successively $n-1$ compositions $R_{1} \circ R_{2},\left(R_{1} \circ R_{2}\right) \circ R_{3}, \ldots$, $\left(\ldots\left(R_{1} \circ R_{2}\right) \cdots \circ R_{n-1}\right) \circ R_{n}$.

Each composition construction can be carried out in $|\mathscr{P}|^{4}$ steps. Indeed, given two relations $A, B \subseteq \mathscr{P} \times$ $\mathscr{P}$, to compute their composition $A \circ B$ requires for each pair $(a, b) \in A$ to find all pairs $(c, d) \in B$ such that $b=c$. Finally, to check whether the intersection of $R_{1} \circ \cdots \circ R_{n}$ with $I$ is non-empty requires at most $|\mathscr{P}|$ steps.

So to answer the original question takes $O\left(n \cdot|\mathscr{P}|^{4}\right)$ time.

Note that this proof applies to any strategic game in which there is a reordering of players $\pi(1), \ldots, \pi(n)$ such that the payoff of player $\pi(i)$ depends only on his strategy and the strategy chosen by player $\pi(i \ominus i)$.

It is worthwhile to note that for the case of simple cycles, the existence of Nash equilibrium in the associated social network game does not imply that the game is weakly acyclic.

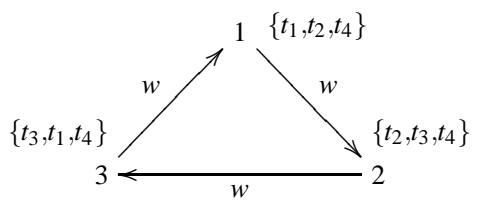

(a)

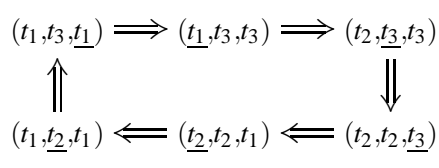

(b)

Figure 3: A simple cycle and an infinite improvement path

Example 5. Consider the network in Figure 3(a) which is a modification of the network in Figure 2 We add a new product $t_{4}$ to the product set of all the nodes $i$ with $\theta\left(i, t_{4}\right)>r_{1}$. We also assume that $w-\theta\left(i, t_{4}\right)>-r_{2}$. Then the joint strategy $\left(t_{4}, t_{4}, t_{4}\right)$ is a Nash equilibrium. However, Figure 3 (b) shows the unique improvement path starting in $\left(t_{1}, t_{3}, t_{1}\right)$ which is infinite. For each joint strategy in the figure, we underline the strategy that is not a best response. This shows that the game is not weakly acyclic.

In Section 4 we shall study the complexity of checking whether a social network game is weakly acyclic.

\subsection{Arbitrary social networks}

In this section we establish two results which show that deciding whether a social network has a Nash equilibrium is computationally hard.

Theorem 6. Deciding whether for a social network $\mathscr{S}$ the game $\mathscr{G}(\mathscr{S})$ has a Nash equilibrium is NPcomplete.

To prove the result we first construct another example of a social network game with no Nash equilibrium and then use it to determine the complexity of the existence of Nash equilibria.

Example 7. Consider the network given in Figure 4, where the product set of each agent is marked next to the node denoting it and the weights are labels on the edges. Nodes with a unique product in the product set is simply represented by the product. 


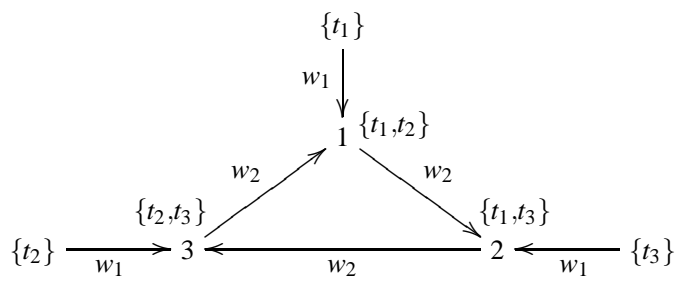

Figure 4: A network with no Nash equilibrium

We assume that each threshold is a constant $\theta$, where $\theta<w_{1}<w_{2}$. So it is more profitable to a player residing on a triangle to adopt the product adopted by his neighbour residing on a triangle than by the other neighbour.

The game associated with this network has no Nash equilibrium. It suffices to analyze the joint strategies involving nodes 1,2 and 3 since the other nodes have exactly one product in their product sets. Here we provide a listing of all such joint strategies, where we underline the strategy that is not a best response to the choice of other players: $\left(\underline{t_{1}}, t_{1}, t_{2}\right),\left(t_{1}, t_{1}, \underline{t_{3}}\right),\left(t_{1}, t_{3}, \underline{t_{2}}\right),\left(t_{1}, \underline{t_{3}}, t_{3}\right),\left(t_{2}, \underline{t}_{1}, t_{2}\right),\left(t_{2}, \underline{t_{1}}, t_{3}\right)$, $\left(t_{2}, t_{3}, \underline{t_{2}}\right),\left(\underline{t_{2}}, t_{3}, t_{3}\right)$. In contrast, what will be of relevance in a moment, if we replace $\left\{\overline{t_{1}}\right\}$ by $\left\{t_{1}^{\prime}\right\}$, then the corresponding game has a Nash equilibrium, namely the joint strategy corresponding to the triple $\left(t_{2}, t_{3}, t_{3}\right)$.

Proof of Theorem 6; As in [1], to show NP-hardness, we use a reduction from the NP-complete PARTITION problem, which is: given $n$ positive rational numbers $\left(a_{1}, \ldots, a_{n}\right)$, is there a set $S$ such that $\sum_{i \in S} a_{i}=\sum_{i \notin S} a_{i}$ ? Consider an instance $I$ of PARTITION. Without loss of generality, suppose we have normalised the numbers so that $\sum_{i=1}^{n} a_{i}=1$. Then the problem instance sounds: is there a set $S$ such that $\sum_{i \in S} a_{i}=\sum_{i \notin S} a_{i}=\frac{1}{2}$ ?

To construct the appropriate network we employ the networks given in Figure 4 and in Figure 5 where for each node $i \in\{1, \ldots, n\}$ we set $w_{i a}=w_{i b}=a_{i}$, and assume that the thresholds of the nodes $a$ and $b$ are constant and equal $\frac{1}{2}$.

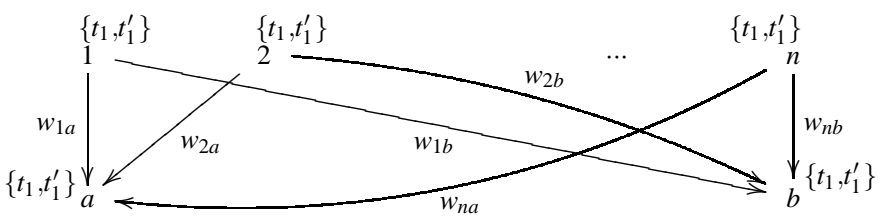

Figure 5: A network related to the PARTITION problem

To finalize the construction we use two copies of the network given in Figure 4, one unchanged and the other in which the product $t_{1}$ is replaced everywhere by $t_{1}^{\prime}$, and construct the desired network $\mathscr{S}$ by identifying with the node marked by $\left\{t_{1}\right\}$ in the network from Figure 4, the node $a$ of the network from Figure 5 and with the node marked by $\left\{t_{1}^{\prime}\right\}$ in the modified version of the network from Figure 4 the node $b$.

Suppose that a solution to the considered instance of the PARTITION problem exists, i.e., for some set $S \subseteq\{1, \ldots, n\}$ we have $\sum_{i \in S} a_{i}=\sum_{i \notin S} a_{i}=\frac{1}{2}$. Consider the game $\mathscr{G}(\mathscr{S})$ and the joint strategy $s$ formed by the following strategies:

- $t_{1}$ assigned to each node $i \in S$ in the network from Figure 5 ,

- $t_{1}^{\prime}$ assigned to each node $i \in\{1, \ldots, n\} \backslash S$ in the network from Figure 5 
- $t_{1}^{\prime}$ assigned to the nodes $a$ and $t_{1}$ to the node $b$,

- $t_{2}$ assigned to node 1 and $t_{3}$ assigned to the nodes 2, 3 in both versions of the networks from Figure 4

- $t_{2}$ and $t_{3}$ assigned respectively to the nodes marked by $\left\{t_{2}\right\}$ and $\left\{t_{3}\right\}$.

We claim that $s$ is a Nash equilibrium. Consider first the player (i.e., node) $a$. The accumulated weight of its neighbours who chose strategy $t_{1}^{\prime}$ is $\frac{1}{2}$. Therefore, the payoff for $a$ in the joint strategy $s$ is 0 . The accumulated weight of its neighbours who chose strategy $t_{1}$ is $\frac{1}{2}$, as well. Therefore $t_{1}^{\prime}$ is indeed a best response for player $a$ as both strategies yield the same payoff. For the same reason, $t_{1}$ is a best response for player $b$. The analysis for the other nodes is straightforward.

Conversely, suppose that a strategy profile $s$ is a Nash equilibrium in $\mathscr{G}(\mathscr{S})$. From Example 7 it follows that $s_{a}=t_{1}^{\prime}$ and $s_{b}=t_{1}$. This implies that $t_{1}^{\prime}$ is a best response of node $a$ to $s_{-a}$ and therefore $\sum_{i \in\{1, \ldots, n\} \mid s_{i}=t_{1}^{\prime}} w_{i a} \geq \sum_{i \in\{1, \ldots, n\} \mid s_{i}=t_{1}} w_{i a}$. By a similar reasoning, for node $b$ we have $\sum_{i \in\{1, \ldots, n\} \mid s_{i}=t_{1}} w_{i b} \geq$ $\sum_{i \in\{1, \ldots, n\} \mid s_{i}=t_{1}^{\prime}} w_{i b}$. Since $\sum_{i=1}^{n} a_{i}=1$ and for $i \in\{1, \ldots, n\}, w_{i a}=w_{i b}=a_{i}$, and $s_{i} \in\left\{t_{1}, t_{1}^{\prime}\right\}$ we have for $S:=\left\{i \in\{1, \ldots, n\} \mid s_{i}=t_{1}\right\}, \sum_{i \in S} a_{i}=\sum_{i \notin S} a_{i}$. In other words, there exists a solution to the considered instance of the partition problem.

Theorem 8. For a network $\mathscr{S}$ whose underlying graph has no source nodes, deciding whether the game $\mathscr{G}(\mathscr{S})$ has a Nash equilibrium is NP-complete.

Proof. The proof extends the proof of the above theorem. Given an instance of the PARTITION problem we use the following modification of the network. We 'twin' each node $i \in\{1, \ldots, n\}$ in Figure 5 with a new node $i^{\prime}$ with the product set $\left\{t_{1}, t_{1}^{\prime}\right\}$, by adding the edges $\left(i, i^{\prime}\right)$ and $\left(i^{\prime}, i\right)$. We also 'twin' nodes marked $\left\{t_{2}\right\}$ and $\left\{t_{3}\right\}$ in Figure 4 with new nodes with the product set $\left\{t_{2}\right\}$ and $\left\{t_{3}\right\}$ respectively. Additionally, we choose the weights on the new edges $w_{i i^{\prime}}, w_{i^{\prime} i}$ and the corresponding thresholds so that when $i$ and $i^{\prime}$ adopt a common product, their payoff is positive. Then the underlying graph of the resulting network does not have any source nodes and the above proof remains valid for this new network.

\section{Weakly acyclic games}

In this section we study the complexity of checking whether a social network game is weakly acyclic. We establish two results that are analogous to the ones established in [15] for the case of social networks in which the nodes may decide not to choose any product. The proofs are based on similar arguments though the details are different.

Theorem 9. For an arbitrary network $\mathscr{S}$, deciding whether the game $\mathscr{G}(\mathscr{S})$ is weakly acyclic is co-NP hard.

Proof. We again use an instance of the PARTITION problem in the form of $n$ positive rational numbers $\left(a_{1}, \ldots, a_{n}\right)$ such that $\sum_{i=1}^{n} a_{i}=1$. Consider the network given in Figure 6 For each node $i \in\{1, \ldots, n\}$ we set $P(i)=\left\{t_{1}, t_{2}\right\}$. The product set for the other nodes are marked in the figure. As before, we set $w_{i a}=w_{i b}=a_{i}$.

Since for all $i \in\{1, \ldots, n\}, a_{i}$ is rational, it has the form $a_{i}=\frac{l_{i}}{r_{i}}$. Let $\tau=\frac{1}{2 \cdot r_{1} \cdot \ldots \cdot r_{n}}$. The following property holds.

Property 1. Given an instance $\left(a_{1}, \ldots, a_{n}\right)$ of the PARTITION problem and $\tau$ defined as above, for all $S \subseteq\{1, \ldots, n\}$ 


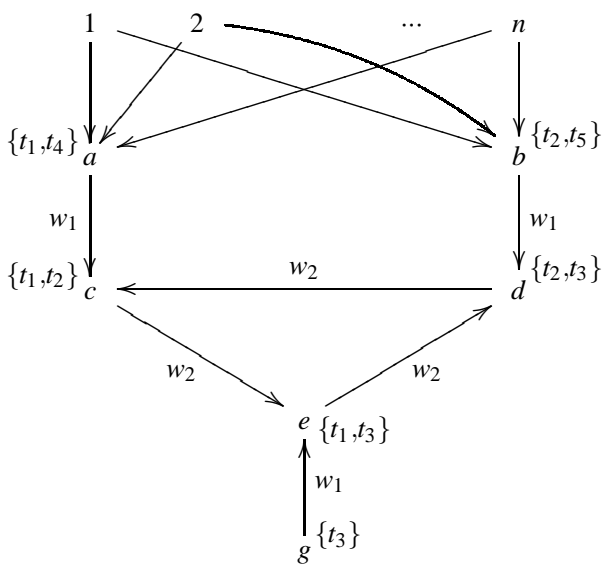

Figure 6: A network related to weakly acyclic games

(i) if $\sum_{i \in S} a_{i}<\frac{1}{2}$, then $\sum_{i \in S} a_{i} \leq \frac{1}{2}-\tau$,

(ii) if $\sum_{i \in S} a_{i}>\frac{1}{2}$, then $\sum_{i \in S} a_{i} \geq \frac{1}{2}+\tau$.

Proof. By definition, each $a_{i}$ and $\frac{1}{2}$ is a multiple of $\tau$. Thus $\sum_{i \in S} a_{i}=x \cdot \tau$ and $\frac{1}{2}=y \cdot \tau$ where $x$ and $y$ are integers.

(i) If $x \cdot \tau<y \cdot \tau$, then $x \cdot \tau \leq(y-1) \cdot \tau$. Therefore $\sum_{i \in S} a_{i} \leq \frac{1}{2}-\tau$.

The proof of $(i i)$ is analogous.

Note that given $\left(a_{1}, \ldots, a_{n}\right), \tau$ can be defined in polynomial time. Let the thresholds be defined as follows: $\theta\left(a, t_{1}\right)=\theta\left(b, t_{2}\right)=\frac{1}{2}$ and $0<\theta\left(a, t_{4}\right)=\theta\left(b, t_{5}\right)<\tau$. The threshold for nodes $c, d$ and $e$ is a constant $\theta_{1}$ such that $\theta_{1}<w_{1}<w_{2}$. Thus, like in the network in Figure 4 it is more profitable to a player residing on a triangle to adopt the product adopted by his neighbour residing on a triangle than by the other neighbour.

Suppose that a solution to the considered instance of the PARTITION problem exists. That is, for some set $S \subseteq\{1, \ldots, n\}$ we have $\sum_{i \in S} a_{i}=\sum_{i \notin S} a_{i}=\frac{1}{2}$. In the game $\mathscr{G}(\mathscr{S})$, take the joint strategy $s$ formed by the following strategies:

- $t_{1}$ assigned to each node $i \in S$ and the nodes $a$ and $c$,

- $t_{2}$ assigned to each node $i \in\{1, \ldots, n\} \backslash S$ and the nodes $b$ and $d$,

- $t_{3}$ assigned to the nodes $e$ and $g$.

Any improvement path that starts in this joint strategy will not change the strategies assigned to the nodes $a, b$ and $g$. So if such an improvement path terminates, it produces a Nash equilibrium in the game associated with the network given in Figure 4 of Example 7 But we argued that this game does not have a Nash equilibrium. Consequently, there is no finite improvement path in the game $\mathscr{G}(\mathscr{S})$ that starts in the above joint strategy and therefore $\mathscr{G}(\mathscr{S})$ is not weakly acyclic.

Now suppose that the considered instance of the PARTITION problem does not have a solution. Then we show that the game $\mathscr{G}(\mathscr{S})$ is weakly acyclic. To this end, we order the nodes of $\mathscr{S}$ as follows (note the positions of the nodes $c, d$ and $e$ ): $1,2, \ldots, n, g, a, b, c, e, d$. Given a joint strategy, consider an improvement path in which at each step the first node in the above list that did not select a best response 
switches to a best response. After at most $n$ steps the nodes $1,2, \ldots, n$ all selected a product $t_{1}$ or $t_{2}$. Let $s$ be the resulting joint strategy.

First suppose that $\sum_{i \in\{1, \ldots, n\} \mid s_{i}=t_{1}} w_{i a}>\frac{1}{2}$. This implies that $\sum_{i \in\{1, \ldots, n\} \mid s_{i}=t_{2}} w_{i b}<\frac{1}{2}$. By Property 1 $\sum_{i \in\{1, \ldots, n\} \mid s_{i}=t_{2}} w_{i b} \leq \frac{1}{2}-\tau$. The payoff of the node $b$ depends only on the choices made by the source nodes $1,2, \ldots, n$, so we have $p_{b}\left(t_{2}, s_{-b}\right) \leq-\tau$. Since $\theta\left(b, t_{5}\right)<\tau$, we also have $p_{b}\left(t_{5}, s_{-b}\right)>-\tau$ and therefore $t_{5}$ is a best response for node $b$. Let $s^{b}$ be the resulting strategy in which node $b$ selects $t_{5}$. Consider the prefix of $\xi$ starting at $s^{b}$ (call it $\xi^{b}$ ). We argue that in $\xi^{b}, t_{2}$ is never a better response for node $d$. Suppose that $s_{d}^{b}=t_{3}$. We have the following two cases:

- $s_{e}^{b}=t_{3}$ : then $p_{d}\left(s^{b}\right)=w_{2}-\theta_{1}$ and so $t_{3}$ is the best response for node $d$.

- $s_{e}^{b}=t_{1}$ : then $p_{d}\left(s^{b}\right)=-\theta_{1}$ and if node $d$ switches to $t_{2}$ then $p_{d}\left(t_{2}, s_{-b}^{b}\right)=-\theta_{1}$ (since $s_{b}^{b}=t_{5}$ ). Thus $t_{2}$ is not a better response.

Using the above observation, we conclude that there exists a suffix of $\xi^{b}$ (call it $\xi^{d}$ ) such that node $d$ never chooses $t_{2}$. This means that in $\xi^{d}$ the unique best response for node $c$ is $t_{1}$ and for node $e$ is $t_{1}$. This shows that $\xi^{d}$ is finite and hence $\xi$ is finite, as well.

The case when $\sum_{i \in\{1, \ldots, n\} \mid s_{i}=t_{2}} w_{i b}>\frac{1}{2}$ is analogous with all improvement paths terminating in a joint strategy where node $a$ chooses $t_{4}$ and node $c$ chooses $t_{2}$.

Theorem 10. For a network $\mathscr{S}$ whose underlying graph has no source nodes, deciding whether the game $\mathscr{G}(\mathscr{S})$ is weakly acyclic is co-NP hard.

Proof. The proof extends the proof of the above theorem. Given an instance of the PARTITION problem we use the following modification of the network given in Figure 6 We 'twin' each node $i \in\{1, \ldots, n\}$ with a new node $i^{\prime}$, also with the product set $\left\{t_{1}, t_{2}\right\}$, by adding the edges $\left(i, i^{\prime}\right)$ and $\left(i^{\prime}, i\right)$. We also 'twin' the node $g$ with a new node $g^{\prime}$, also with the product set $\left\{t_{3}\right\}$, by adding the edges $\left(g, g^{\prime}\right)$ and $\left(g^{\prime}, g\right)$. Additionally, we choose the weights $w_{i i^{\prime}}$ and $w_{i^{\prime} i}$ and the corresponding thresholds so that when $i$ and $i^{\prime}$ adopt a common product, their payoff is positive.

Suppose that a solution to the considered instance of the PARTITION problem exists. Then we extend the joint strategy considered in the proof of Theorem 9 by additionally assigning $t_{1}$ to each node $i^{\prime}$ such that $i \in S, t_{2}$ to each node $i^{\prime}$ such that $i \in\{1, \ldots, n\} \backslash S$ and $t_{3}$ to the node $g^{\prime}$. Then, as before, there is no finite improvement path starting in this joint strategy, so $\mathscr{G}(\mathscr{S})$ is not weakly acyclic.

Suppose now that no solution to the considered instance of the PARTITION problem exists. Take the following order of the nodes of $\mathscr{S}$ :

$$
1,1^{\prime}, 2,2^{\prime}, \ldots, n, n^{\prime}, g, g^{\prime}, a, b, c, e, d,
$$

and as in the previous proof, given a joint strategy, we consider an improvement path $\xi$ in which at each step the first node in the above list that did not select a best response switches to a best response.

Note that each node from the list $1,1^{\prime}, 2,2^{\prime}, \ldots, n, n^{\prime}, g, g^{\prime}$ is scheduled at most once. So there exists a suffix of $\xi$ in which only the nodes $a, b, c, e, d$ are scheduled. Using now the argument given in the proof of Theorem 9 we conclude that there exists a suffix of $\xi$ that is finite. This proves that $\mathscr{G}(\mathscr{S})$ is weakly acyclic.

\section{Paradoxes}

In [2] we identified various paradoxes in social networks with multiple products and studied them using the social network games introduced in [14]. Here we carry out an analogous analysis for the case when 
the product selection is obligatory. This qualification, just like in the case of social network games, substantially changes the analysis. We focus on the main four paradoxes that we successively introduce and analyze.

\subsection{Vulnerable networks}

The first one is the following. We say that a social network $\mathscr{S}$ is vulnerable if for some Nash equilibrium $s$ in $\mathscr{G}(\mathscr{S})$, an expansion $\mathscr{S}^{\prime}$ of $\mathscr{S}$ exists such that each improvement path in $\mathscr{G}\left(\mathscr{S}^{\prime}\right)$ leads from $s$ to a joint strategy $s^{\prime}$ which is a Nash equilibrium both in $\mathscr{G}\left(\mathscr{S}^{\prime}\right)$ and $\mathscr{G}(\mathscr{S})$ such that $s>s^{\prime}$. So the newly added product triggers a sequence of changes that unavoidably move the players from one Nash equilibrium to another one that is strictly worse for everybody.

The following example shows that vulnerable networks exist. Here and elsewhere the relevant expansion is depicted by means of a product and the dotted arrow pointing to the relevant node.

Example 11. Consider the directed graph given in Figure 7 in which the product set of each node is marked next to it.

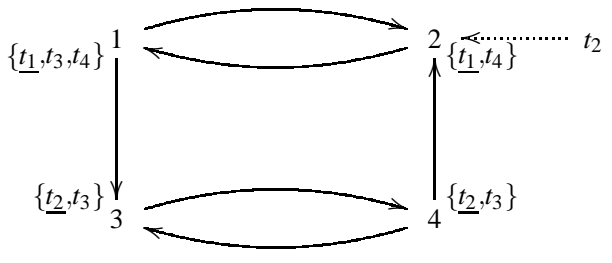

Figure 7: A directed graph

We complete it to the desired social network below. Let ' -' stand for an arbitrary strategy of the relevant player. We stipulate that

$$
\begin{aligned}
& p_{2}\left({ }_{-}, t_{2},{ }_{-}, t_{2}\right)>p_{2}\left(t_{1}, t_{1},_{-},{ }_{-}\right), \\
& p_{1}\left(t_{3}, t_{2},,_{-},-\right)>p_{1}\left(t_{1}, t_{2},,_{-},-\right)>p_{1}\left(t_{4}, t_{2},,_{-},-\right), \\
& p_{3}\left(t_{3},-, t_{3},-\right)>p_{3}\left(-,-, t_{2}, t_{2}\right), \\
& p_{4}\left({ }_{-}, t_{3}, t_{3}\right)>p_{4}\left({ }_{-},-, t_{3}, t_{2}\right), \\
& p_{2}\left({ }_{-}, t_{4},{ }_{-},-\right)>p_{2}\left({ }_{-}, t_{2},{ }_{-}, t_{3}\right) \text {, } \\
& p_{1}\left(t_{4}, t_{4},,_{-},-\right)>p_{1}\left(t_{3},,_{-},,_{-}\right)>p_{1}\left(t_{1}, t_{4},,_{-},{ }_{-}\right) \text {, }
\end{aligned}
$$

so that $2: t_{2}, 1: t_{3}, 3: t_{3}, 4: t_{3}, 2: t_{4}, 1: t_{4}$ is a unique improvement path that starts in $\left(t_{1}, t_{1}, t_{2}, t_{2}\right)$ and ends in $\left(t_{4}, t_{4}, t_{3}, t_{3}\right)$.

Additionally we stipulate that

$p_{1}\left(t_{1}, t_{1},_{-},-\right)>p_{1}\left(t_{4}, t_{4},_{-},-\right)$,

$p_{2}\left(t_{1}, t_{1},_{-},-\right)>p_{2}\left(t_{4}, t_{4},_{-},-\right)$,

$p_{3}\left({ }_{-}, t_{2}, t_{2}\right)>p_{3}\left({ }_{-}, t_{-}, t_{3}, t_{3}\right)$,

$p_{4}\left({ }_{-}, t_{2}, t_{2}\right)>p_{4}\left({ }_{-},-, t_{3}, t_{3}\right)$,

so that $\left(t_{1}, t_{1}, t_{2}, t_{2}\right)>_{s}\left(t_{4}, t_{4}, t_{3}, t_{3}\right)$.

These requirements entail constraints on the weights and thresholds that are for instance realized by $w_{12}=0, w_{21}=0.2, w_{42}=0.3, w_{13}=0.2, w_{34}=0.2, w_{43}=0$,

and

$\theta\left(1, t_{1}\right)=0.2, \theta\left(1, t_{3}\right)=0.1, \theta\left(1, t_{4}\right)=0.3, \theta\left(2, t_{1}\right)=0.1, \theta\left(2, t_{2}\right)=0.3$,

$\theta\left(2, t_{4}\right)=0.2, \theta\left(3, t_{2}\right)=0.1, \theta\left(3, t_{3}\right)=0.2, \theta\left(4, t_{2}\right)=0.1, \theta\left(4, t_{3}\right)=0.2$. 
It is useful to note that in the setup of [2], in which for each player the 'abstain' strategy is allowed, it remains an open problem whether vulnerable networks (called there because of various other alternatives $\forall s$-vulnerable networks) exist.

\subsection{Fragile networks}

Next, we consider the following notion. We say that a social network $\mathscr{S}$ is fragile if $\mathscr{G}(\mathscr{S})$ has a Nash equilibrium while for some expansion $\mathscr{S}^{\prime}$ of $\mathscr{S}, \mathscr{G}\left(\mathscr{S}^{\prime}\right)$ does not. The following example shows that fragile networks exist.

Example 12. Consider the network $\mathscr{S}$ given in Figure 8 , where the product set of each node is marked next to it.

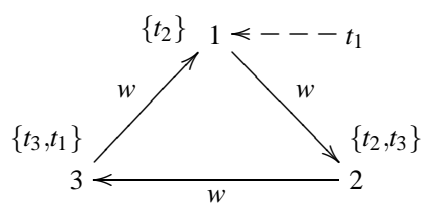

Figure 8: A fragile network

Let the thresholds be defined as follows: $\theta\left(2, t_{2}\right)=\theta\left(3, t_{3}\right)=r_{1}$ and $\theta\left(1, t_{2}\right)=\theta\left(2, t_{3}\right)=\theta\left(3, t_{1}\right)=r_{2}$ where $r_{1}>r_{2}$. We also assume that $w>r_{1}-r_{2}$.

Consider the joint strategy $s$, in which nodes 1,2 and 3 choose $t_{2}, t_{2}$ and $t_{1}$ respectively. It can be verified that $s$ is a Nash equilibrium in $\mathscr{G}(\mathscr{S})$. Now consider the expansion $\mathscr{S}^{\prime}$ of $\mathscr{S}$ in which product $t_{1}$ is added to the product set of node 1 and let $\theta\left(1, t_{1}\right)=r_{1}$. Then $\mathscr{S}^{\prime}$ is the network in Example 3 which, as we saw, does not have a Nash equilibrium.

\subsection{Inefficient networks}

We say that a social network $\mathscr{S}$ is inefficient if for some Nash equilibrium $s$ in $\mathscr{G}(\mathscr{S})$, a contraction $\mathscr{S}^{\prime}$ of $\mathscr{S}$ exists such that each improvement path in $\mathscr{G}\left(\mathscr{S}^{\prime}\right)$ starting in $s$ leads to a joint strategy $s^{\prime}$ which is a Nash equilibrium both in $\mathscr{G}\left(\mathscr{S}^{\prime}\right)$ and $\mathscr{G}(\mathscr{S})$ such that $s^{\prime}>s$. We note here that if the contraction was created by removing a product from the product set of node $i$, we impose that any improvement path in $\mathscr{G}\left(\mathscr{S}^{\prime}\right)$, given a starting joint strategy from $\mathscr{G}(\mathscr{S})$, begins by having node $i$ making a choice (we allow any choice from his remaining set of products as an improvement move). Otherwise the initial payoff of node $i$ in $\mathscr{G}\left(\mathscr{S}^{\prime}\right)$ is not well-defined.

Example 13. We exhibit in Figure 9 an example of an inefficient network. The weight of each edge is assumed to be $w$, and we also have the same product-independent threshold, $\theta$, for all nodes, with $w>\theta$.

Consider as the initial Nash equilibrium the joint strategy $s=\left(t_{2}, t_{2}, t_{1}, t_{1}\right)$. It is easy to check that this is indeed a Nash equilibrium, with the payoff equal to $w-\theta$ for all nodes. Suppose now that we remove product $t_{1}$ from the product set of node 3 . We claim that the unique improvement path then leads to the Nash equilibrium in which all nodes adopt $t_{2}$.

To see this, note that node 3 moves first in any improvement path and it has a unique choice, $t_{2}$. Then node 4 moves and necessarily switches to $t_{2}$. This yields a Nash equilibrium in which each node selected $t_{2}$ with the payoff of $2 w-\theta$, which is strictly better than the payoff in $s$. 


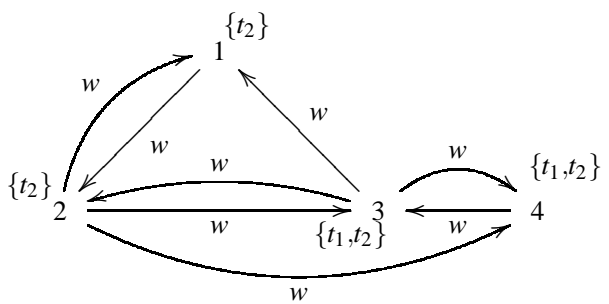

Figure 9: An example of an inefficient network

\subsection{Unsafe networks}

Finally, we analyze the following notion. We call a social network $\mathscr{S}$ unsafe if $\mathscr{G}(\mathscr{S})$ has a Nash equilibrium, while for some contraction $\mathscr{S}^{\prime}$ of $\mathscr{S}, \mathscr{G}\left(\mathscr{S}^{\prime}\right)$ does not. The following example shows that unsafe networks exist.

Example 14. Let $\mathscr{S}_{1}$ be the modification of the network $\mathscr{S}$ given in Figure 2 where node 1 has the product set $\left\{t_{1}, t_{2}, t_{4}\right\}$, where $\theta\left(1, t_{4}\right)<r_{2}$. Then the joint strategy $\left(t_{4}, t_{3}, t_{3}\right)$ is a Nash equilibrium in $\mathscr{G}\left(\mathscr{S}_{1}\right)$. Now consider the contraction $\mathscr{S}_{1}^{\prime}$ of $\mathscr{S}_{1}$ where product $t_{4}$ is removed from node 1 . Then $\mathscr{S}_{1}^{\prime}$ is the network $\mathscr{S}$, which as we saw in Example 3 has no Nash equilibrium.

\section{Conclusions}

In this paper we studied dynamic aspects of social networks with multiple products using the basic concepts of game theory. We used the model of social networks, originally introduced in [1] that we subsequently studied using game theory in [14], [15] and [2].

However, in contrast to these three references the product adoption in this paper is obligatory. This led to some differences. For example, in contrast to the case of [14], a Nash equilibrium does not need to exist when the underlying graph is a simple cycle. Further, in contrast to the setup of [2], we were able to construct a social network that exhibits the strongest form of the paradox of choice. On the other hand, some complexity results, namely the ones concerning weakly acyclic games, remain the same as in [14], though the proofs had to be appropriately modified.

\section{References}

[1] K. R. Apt \& E. Markakis (2011): Diffusion in Social Networks with Competing Products. In: Proc. 4th International Symposium on Algorithmic Game Theory (SAGT11), Lecture Notes in Computer Science 6982, Springer, pp. 212-223, doi:10.1007/978-3-642-24829-0_20.

[2] K. R. Apt, E. Markakis \& S. Simon (2013): Paradoxes in Social Networks with Multiple Products. Manuscript, CWI, Amsterdam, The Netherlands. Computing Research Repository (CoRR), http: //arxiv . org/abs/1301.7592.

[3] David Easley \& Jon Kleinberg (2010): Networks, Crowds, and Markets. Cambridge University Press.

[4] D. Fotakis, A. C. Kaporis, T. Lianeas \& P. G. Spirakis (2012): On the Hardness of Network Design for Bottleneck Routing Games. In: SAGT, pp. 156-167, doi:10.1007/978-3-642-33996-7_14.

[5] D. Fotakis, A. C. Kaporis \& P. G. Spirakis (2012): Efficient methods for selfish network design. Theor. Comput. Sci. 448, pp. 9-20, doi:10.1016/j.tcs.2012.04.033.

[6] G. Gigerenzer (2008): Gut Feelings: The Intelligence of the Unconscious. Penguin. Reprint edition. 
[7] M.O. Jackson (2008): Social and Economic Networks. Princeton University Press, Princeton.

[8] M. Kearns, M. Littman \& S. Singh (2001): Graphical models for game theory. In: Proceedings of the 17th Conference in Uncertainty in Artificial Intelligence (UAI '01), Morgan Kaufmann, pp. 253-260.

[9] I. Milchtaich (1996): Congestion Games with Player-Specific Payoff Functions. Games and Economic Behaviour 13, pp. 111-124, doi:10.1006/game.1996.0027.

[10] D. Monderer \& L. S. Shapley (1996): Potential Games. Games and Economic Behaviour 14, pp. 124-143, doi:10.1006/game.1996.0044.

[11] S. Morris (2000): Contagion. The Review of Economic Studies 67(1), pp. 57-78, doi:10.1111/1467-937X. 00121.

[12] N. Nisan, T. Roughgarden, É. Tardos \& V. J. Vazirani, editors (2007): Algorithmic Game Theory. Cambridge University Press.

[13] B. Schwartz (2005): Paradox of Choice: Why More Is Less. Harper Perennial.

[14] S. Simon \& K. R. Apt (2012): Choosing Products in Social Networks. In: Proc. 8th International Workshop on Internet and Network Economics (WINE), Lecture Notes in Computer Science 7695, Springer, pp. 100113, doi:10.1007/978-3-642-35311-6_8.

[15] S. Simon \& K. R. Apt (2013): Social Network Games. Journal of Logic and Computation, doi:10.1093/ logcom/ext012. To appear.

[16] H. Peyton Young (1993): The evolution of conventions. Econometrica 61(1), pp. 57-84, doi:10.2307/ 2951778. 